\title{
Candidúria: uma abordagem clínica e terapêutica
}

\author{
Candiduria: a clinical and therapeutic approach
}

Arnaldo Lopes Colombo ${ }^{1}$ Thaís Guimarães ${ }^{1,2}$

\begin{abstract}
RESUMO
O achado laboratorial de candidúria traz dilemas em relação a sua interpretação visto que pode refletir uma amplitude de possibilidades clínicas, incluindo colonização, infecção urinária alta ou doença sistêmica por Candida spp. Neste artigo, abordaremos a epidemiologia, o diagnóstico e a terapêutica da candidúria em diversos cenários clínicos, incluindo pacientes transplantados renais. De forma prática e para efeito de abordagem terapêutica, a interpretação do achado de candidúria é baseada na presença de dados clínicos e epidemiológicos Quando necessária, a terapêutica antifúngica para os casos de candidúria pode ser realizada com: anfotericina B sistêmica, anfotericina B tópica (irrigação vesical) ou fluconazol. A coleta de hemoculturas deve ser indicada em pacientes com candidúria sob risco para desenvolvimento de candidíase hematogênica. A retirada da sonda vesical de demora deve ser considerada sempre que possivel, pois reduz a possibilidade de persistência ou recorrência da infecção urinária por Candida spp.
\end{abstract}

Palavras-chaves: Candidúria. Infecção hospitalar. Antifúngicos. Candida.

\begin{abstract}
Candiduria remains a controversial issue for clinicians once that it may represent a broad variety of possibilities including colonization, local or systemic infection. We will discuss the epidemiology, diagnosis and treatment of candiduria in different settings of patients, including renal transplant recipients. Definitions on therapy are mostly based on epidemiological and clinical data. Once antifungal therapy is required the following antifungal treatment may be used: intravenous amphotericin B, bladder irrigation with amphotericin B or fluconazole. Blood cultures may be required in patients with candiduria and high risk for developing hematogenous infection. Removal of the urinary catheter must be considered in order to avoid persistent candiduria and recurrence.
\end{abstract}

Key-words: Candiduria. Nosocomial infection. Antifungal drugs. Candida.

\section{EPIDEMIOLOGIA}

O termo candidúria, que não necessariamente envolve a presença de sinais e/ou sintomas de infecção urinária, pode ser definido como o crescimento de Candida sp em culturas de urina coletadas por técnicas apropriadas. Trata-se de evento muito freqüente entre pacientes expostos a fatores de risco, sendo que até $20 \%$ de pacientes hospitalizados podem apresentar candidúria ao longo de sua internação, particularmente pacientes de unidade de terapia intensiva $a^{611141726}$. Este achado laboratorial traz dilemas em relação a sua interpretação, visto que pode corresponder a simples contaminação da coleta de urina até candidúria assintomática, cistite ou pielonefrite, candidíase renal primária, bola fúngica ureteropélvica ou candidíase disseminada com manifestação renal ${ }^{1637}$.
Entre pacientes hospitalizados, vários fatores têm sido reconhecidos como relacionados a um maior risco para 0 desenvolvimento de candidúria: idade avançada, sexo feminino, antibioticoterapia de amplo espectro, o uso de corticosteróides e imunossupressores, presença de anormalidades do trato urinário, diabetes, sondagem vesical de demora, pós-operatório de cirurgias de grande porte e doenças malignas 1216172039 (Tabela 1). Importante observar que a diabetes é a doença de base mais frequentemente encontrada nestes pacientes, condição esta associada a neuropatia autonômica que pode evoluir com retenção de urina, episódios de infecção urinária bacteriana (uso de antibióticos recorrente) e necessidade de sondagens de alívio. Em relação à sondagem vesical de demora, o tempo de uso deste dispositivo parece ter relação direta com o risco de desenvolvimento de candidúria. Em estudo conduzido por

\footnotetext{
1. Disciplina de Doenças Infecciosas, Universidade Federal de São Paulo, São Paulo, SP. 2. Hospital do Servidor Público Estadual de São Paulo, São Paulo, SP. Endereço para correspondência: Prof. Arnaldo Lopes Colombo. R. Dr. Diogo de Faria 822, Vila Clementino, 04037-002 São Paulo, SP

Telefax: $55115081-3240$

e-mail: colomboal@terra.com.br

Recebido para publicação em 8/3/2007

Aceito em 4/6/2007
} 
Tabela 1 - Fatores associados à candidúria.

Condições associadas
Diabetes mellitus
Doenças malignas
Doença prévia do trato urinário
Idade avançada
Pós-operartório de cirurgias de grande porte
Sexo feminino
Sondagem vesical
Uso de antibioticoterapia de amplo espectro
Uso de corticóides e imunossupresssores

Harmory e Wenzel, durante os anos 70, observou-se que pacientes com candidúria apresentavam uma média de 12 dias de uso de cateter urinário, contra 5 dias no grupo controle ${ }^{12}$.

Em casuísticas de infecções urinárias por leveduras, Candida albicans tem sido considerada a espécie mais comumente isolada do trato urinário, sendo responsável por cerca de $50 \%$ a $70 \%$ dos episódios, seguida por Candida glabrata em 5 a 33 \% e outras espécies de leveduras não-albicans que têm sido relatadas em 8 a $28 \%$ dos $\operatorname{casos}^{8161720}$. No estudo de Kauffman e cols ${ }^{17}$, onde um total de 861 episódios de candidúria em pacientes hospitalizados foi avaliado, Candida albicans respondeu por $52 \%$ dos isolados, seguido por Candida glabrata e Candida tropicalis ${ }^{16}$ (Tabela 2).

Tabela 2 - Etiologia de 861 episódios de candidúria em casuística de hospitais nos EUA.

\begin{tabular}{lcc}
\hline Agentes etiológicos & Número & Percentagem \\
\hline Candida albicans & 446 & 52,0 \\
Candida glabrata & 134 & 16,0 \\
Candida tropicalis & 68 & 8,0 \\
Candida parapsilosis & 35 & 4,0 \\
Candida krusei & 9 & 1,0 \\
Outros & 169 & 19,0 \\
\hline
\end{tabular}

Apesar do predomínio de Candida albicans, tem havido um aumento na incidência de espécies de leveduras não-albicans como agentes de infecção do trato urinário (ITU), incluindo: Candida glabrata, Candida tropicalis, Candida parapsilosis, Candida krusei, Candida lusitaniae e Candida guilliermondi.

Casuísticas do Brasil confirmam que as três espécies mais prevalentes isoladas de urina em pacientes hospitalizados são: Candida albicans, Candida tropicalis e Candida glabrata. Estes estudos demonstram prevalências de 35,5 a 70\% para Candida albicans; 4,6 a 52,5\% para Candida tropicalis e 7 a 8,8\% para Candida glabrata ${ }^{382729}$ (Tabela 3).

A maioria dos microorganismos que causa infecção urinária relacionada a cateter vesical tem origem no trato gastrointestinal dos pacientes, sendo necessário lembrar que até 30\% das mulheres sadias podem apresentar colonização vulvovaginal persistente por Candida $\mathrm{sp}^{21}$. Após a utilização de sondagem vesical de demora, com o passar do tempo, há um aumento da colonização da região periuretral, particularmente na interface cateter - mucosa, sendo que os microorganismos fazem uso da superfície do cateter para ascender até a bexiga. Em uma menor parcela, estes microorganismos podem ser provenientes do
Tabela 3 - Etiologia dos episódios de candidúria em casuísticas de bospitais no Brasil 182729 .

\begin{tabular}{|c|c|c|c|c|c|c|c|c|}
\hline \multirow[t]{2}{*}{ Agente Etiológico } & \multicolumn{2}{|c|}{ Binelli, 2006} & \multicolumn{2}{|c|}{ Kobayashi, 2004} & \multicolumn{2}{|c|}{ Oliveira, 2001} & \multicolumn{2}{|c|}{ Passos, 2005} \\
\hline & $\mathrm{n}^{0}$ & $\%$ & $\mathrm{n}^{\mathrm{o}}$ & $\%$ & $\mathrm{n}^{\underline{0}}$ & $\%$ & $\mathrm{n}^{\underline{0}}$ & $\%$ \\
\hline Candida albicans & 12 & 52,2 & 16 & 35,5 & 36 & 35,6 & 30 & 70 \\
\hline Candida tropicalis & 10 & 43,5 & 10 & 22,2 & 53 & 52,5 & 2 & 4,6 \\
\hline Candida glabrata & - & - & 4 & 8,8 & 8 & 7,9 & 3 & 7 \\
\hline Candida parapsilosis & - & - & 5 & 11,1 & 3 & 3 & 2 & 4,6 \\
\hline Candida kefyr & - & - & - & - & - & - & 2 & 4,6 \\
\hline Candida famata & - & - & - & - & 1 & 1 & 3 & 7 \\
\hline Candida krusei & - & - & 3 & 6,6 & - & - & 1 & 2,2 \\
\hline Candida guilliermondii & - & - & 2 & 4,4 & - & - & - & - \\
\hline Candida sp & 1 & 4,3 & 5 & 11,1 & - & - & - & - \\
\hline Total & & & & & & 01 & & 43 \\
\hline
\end{tabular}

ambiente hospitalar animado ou inanimado adquirido através das mãos dos profissionais de saúde que manuseiam inadequadamente o sistema de sondagem vesical ou mesmo através da infusão de produtos contaminados ${ }^{61130}$.

\section{DILEMAS DIAGNÓSTICOS E VARIÁVEIS DETERMINANTES DA ABORDAGEM TERAPÊUTICA}

Vários autores têm demonstrado que o achado de candidúria em populações de baixo risco, indivíduos sadios e assintomáticos é raro. Nesta população, o encontro de Candida na urina reflete, na maioria das vezes, coleta ou processamento inadequado da amostra, com conseqüente contaminação da cultura ${ }^{11}{ }^{1620}$. Em pacientes expostos a fatores de risco para infecção urinária por Candida , o achado de candidúria pode significar colonização ou infecção. Nestes pacientes, a contagem de colônias é muito variável e diretamente dependente da metodologia utilizada para a coleta de material. Sendo assim, o isolamento de Candida na urina pode ocorrer mesmo na ausência de doença, sendo que existe grande controvérsia sobre a valorização da contagem de colônias obtida em cultura, procedimento este com baixa especificidade e sensibilidade na diferenciação entre pacientes colonizados e infectados por este agente ${ }^{25} 35$.

Alguns autores sugerem que exista maior relação entre candidúria e infecção urinária quando a contagem de colônias na cultura de urina atinge valores da ordem de 1.000 a $10.000 \mathrm{UFC} / \mathrm{mL}$. Entretanto, contagens inferiores podem ser encontradas em pacientes com infecção do trato urinário (ITU) por Candida, particularmente nos casos de pielonefrite adquirida por via hematogênica decorrente de candidíase sistêmica, onde os rins funcionam como filtro e podem refletir contagens baixas na urina. Havendo envolvimento renal como complicação de fungúria, há formação de microabcessos no córtex renal, com penetração das leveduras nos túbulos proximais e conseqüente excreção na urina. Neste sentido, não há consenso entre os autores sobre o valor de corte específico para interpretação de culturas quantitativas de urina, no sentido de reconhecimento de pacientes com infecção urinária alta ou baixa ${ }^{1278111723}$. 
Uma vez que a cultura quantitativa de urina tem valor limitado, outros parâmetros têm sido utilizados para auxiliar o diagnóstico. Na presença de infecção por Candida, o sedimento urinário usualmente contém hemácias e leucócitos, assim como leveduras, pseudo-hifas e debris nécróticos. Entretanto, a ausência de alteração no sedimento urinário não elimina a possibilidade de infecção fúngica, sendo algo freqüente nos casos onde a candidúria é secundária a infecção sistêmica² .

Avaliando os trabalhos mencionados neste artigo, fica claro que apesar de todo progresso nas técnicas convencionais e moleculares para o diagnóstico das infecções fúngicas, 0 diagnóstico laboratorial de infecção urinária por Candida permanece controverso. Para efeito de vigilância das comissões de controle de infecção hospitalar a definição operacional de infecção urinária por Candida mais utilizada é a mesma definição utilizada para infecção bacteriana, ou seja, presença de mais de 100.000UFC/mL de Candida sp em pacientes sintomáticos ${ }^{10}$.

De forma prática, a escolha da abordagem terapêutica para pacientes com candidúria é realizada caso a caso, onde o clínico deve ponderar sua conduta tendo por base a análise de dados clínicos e epidemiológicos que permitam classificar o paciente em uma das seguintes condições: 1) previamente são, sem fatores de risco para candidúria; 2) predisposto à candidúria com candidíase disseminada improvável; ou 3) predisposto à candidúria com provável disseminação sistêmica ${ }^{7} 20$.

Em pacientes assintomáticos, sem fatores de risco para candidíase invasiva, a maior probabilidade é que se trate de contaminação. Na presença de candidúria em pacientes com risco para ITU sem evidência clínica ou sinais de disseminação da infecção, devem ser consideradas as possibilidades de colonização ou infecção localizada. Finalizando, em pacientes críticos, geralmente submetidos à ventilação mecânica e internados em UTI por longos períodos, com síndrome infecciosa persistente apesar de antibioticoterapia de largo espectro, há possibilidade de a candidúria ser secundária à doença fúngica sistêmica. A candidúria não deve ser ignorada em pacientes sépticos, pois pode ser o primeiro sinal de infecção sistêmica ${ }^{72}$.

Apesar de candidúria constituir achado frequiente entre pacientes hospitalizados, apenas uma pequena parcela destes pacientes evoluem para infecção fúngica sistêmica. No estudo multicêntrico realizado por Kauffman e cols ${ }^{17}$ onde mais de 800 pacientes foram avaliados, apenas 1\% deles evoluíram com candidemia ${ }^{17}$. Em outra série de casos avaliada por Sobel e cols ${ }^{36}$, nenhum paciente com candidúria evoluiu para doença disseminada ${ }^{36}$. Recentemente, Binelli e cols ${ }^{3}$ realizaram estudo caso-controle a fim de verificar se a candidúria estava associada com a ocorrência de candidemia. Neste estudo, a análise microbiológica das espécies de Candida sp isoladas na urina de pacientes que desenvolveram candidemia demonstrou que em $43 \%$ (10/24) dos casos a espécie isolada na urina foi diferente da espécie isolada do sangue, sendo que a análise molecular realizada nos 14 pares concordantes demonstrou diferença em 2 casos de Candida tropicalis. Globalmente, houve diferenças inter e/ou intra-espécies em 52\% das amostras colhidas no sangue e na urina ${ }^{3}$. Estes dados corroboram o conceito de que a infecção do trato urinário baixo é raramente fonte de fungemia ${ }^{3}{ }^{23}$.

\section{RELEVÂNCIA DA IDENTIFICAÇÃO DO FUNGO QUANTO À ESPÉCIE}

0 diagnóstico correto das espécies envolvidas nas infecções fúngicas tem interesse não só epidemiológico, mas também clínico. A história natural destas infecções, bem como sua resposta à terapêutica com azóis ou anfotericina $\mathrm{B}$, variam em função das várias espécies relacionadas. Da mesma forma, o reconhecimento de surtos por determinado microorganismo só é possível mediante a identificação correta do agente quanto à espécie assim como a posterior análise de marcadores moleculares ${ }^{13} 31$.

Sob o ponto de vista terapêutico, as espécies de leveduras não-albicans caracterizam-se por apresentar particularidades em relação à sua sensibilidade a diversos antifúngicos. Sendo assim, em estudo de susceptibilidade dos microorganismos, tem sido relatada a ocorrência de resistência primária ou secundária à anfotericina $\mathrm{B}$ em amostras de Candida lusitaniae e Trichosporon spp ${ }^{7}$.

Da mesma forma, acredita-se que, em parcela significativa dos casos, os isolados de Candida não-albicans são menos sensíveis a azólicos quando comparados a amostras de Candida albicans. Relatos de falhas na profilaxia e tratamento com fluconazol têm sido registrados com espécies intrinsecamente menos sensíveis a esta droga, quais sejam: Candida glabrata, Candida krusei e Trichosporon sp. Estas informações enfatizam a importância da identificação acurada das espécies de Candida e o estudo da sua prevalência no ambiente hospitalar, no sentido de aperfeiçoar as condutas de terapêutica empírica ou dirigida a infecções fúngicas invasivas ${ }^{32}$.

\section{QUANDO SOLICITAR TESTES DE SENSIBILIDADE A DROGAS ANTIFÚNGICAS}

Como reflexo do maior interesse das comunidades médica e científica pela padronização de testes de sensibilidade, em 1982, a entidade responsável pela normatização de técnicas de laboratório clínico nos Estados Unidos, o Comitee for Laboratory Standard Institute (CLSI), designou um subcomitê para padronizar os testes de susceptibilidade de fungos a drogas antimicóticas.

o CLSI, numa primeira etapa, deu prioridade a trabalhos envolvendo leveduras. Como parte da estratégia para obter método com alta reprodutibilidade de resultados, vários estudos multicêntricos foram realizados permitindo a padronização das condições técnicas para a execução da macrodiluição e microdiluição em caldo como métodos de referência para estes ensaios. Ambos os métodos têm sido empregados por diversos laboratórios de referência em Micologia Médica comprovando não só a alta reprodutibilidade de seus resultados como boa correlação clínico-laboratorial ${ }^{4}{ }^{38}$.

Baseados em estudos de farmacocinética de antifúngicos bem como nos resultados obtidos na terapêutica de infecções por Candida spp com triazólicos, o CLSI sugeriu valores de breakpoints para interpretação dos resultados de concentrações inibitórias mínimas obtidas com fluconazol, itraconazol, 5-fluorocitosina e voriconazol ${ }^{24}$. 
A utilização destes métodos tem permitido a documentação do perfil de sensibilidade a todas as drogas disponíveis comercialmente, sendo reconhecida a ocorrência de resistência de Candida spp a azólicos ${ }^{32}$.

Tendo em vista estas informações, sugerimos a realização de testes de sensibilidade os antifúngicos para amostras de leveduras provenientes de pacientes hospitalizados que fizeram uso de antifúngico profilático ou terapêutico por longos períodos, nos casos de infecção fúngica documentada após uso prévio empírico ou profilático de azólicos, bem como nos casos de infecção por Candida não albicans onde o clínico pretenda utilizar terapêutica com azólicos.

\section{TERAPÊUTICA}

Antes de iniciar a terapêutica específica, o clínico deve responder às seguintes questões:

As culturas foram colhidas de forma adequada? Havendo dúvida sobre as condições técnicas da coleta de material, esta deve ser repetida dentro das normas específicas padronizadas. Esta conduta é particularmente importante quando a cultura positiva pertence à paciente ambulatorial sem fator de risco estabelecido para ITU por fungos ${ }^{8}$.

Para cultura de urina, recomenda-se a coleta de jato médio após higienização adequada da glande ou vagina. Em pacientes com sonda vesical de demora, amostra de urina deve ser colhida por punção a ser realizada no local específico do circuito para esta finalidade, sempre precedido por assepsia adequada ${ }^{11}$.

A levedura foi corretamente identificada quanto à espécie? Sempre que o clínico for utilizar antifúngico para tratamento de fungúria é fundamental que o agente etiológico seja identificado quanto à espécie, para auxiliar na escolha do esquema terapêutico.

Pacientes ambulatoriais ou hospitalizados onde há suspeita de colonização do trato urinário ou da sonda vesical, situações em que o médico inicialmente vai apenas abordar os fatores predisponentes para fungúria, sem administrar terapêutica antifúngica ao paciente, a identificação da levedura quanto à espécie é prescindível.

Quais pacientes devem receber antifúngico? Esta é a principal dúvida na maioria dos casos, particularmente no manuseio de pacientes hospitalizados. Conforme ilustrado anteriormente, os dados laboratoriais auxiliam muito pouco 0 clínico nesta decisão. Sendo assim, baseado nos dados clínicos e epidemiológicos do paciente, estes podem ser classificados em três grupos:

a) previamente são, sem fatores de risco para candidúria: pacientes sem doenças de base, não submetidos à sondagem vesical, sem antecedente de uso prévio de corticosteróides e antibióticos não devem receber antifúngicos sistêmicos. Solicitar nova coleta de material e caso confirme a presença de leveduras, investigar a possibilidade de mucosite genital por fungo em vagina ou glande $\mathrm{e}^{20}$ b) predisposto à candidúria com candidíase disseminada improvável: pacientes assintomáticos ambulatoriais ou hospitalizados que foram submetidos à sondagem vesical e/ou outros fatores predisponentes para candidúria não devem receber antifúngicos. A abordagem inicial consiste da retirada dos fatores predisponentes com acompanhamento clínico e laboratorial posterior. Agrande maioria destes pacientes fica livre da candidúria logo após a instituição destas medidas ${ }^{811} 20$. Pacientes com sintomas de cistite e cujo único achado em cultura seja a presença de leveduras devem ser tratados com antifúngicos. 0 guia da Sociedade Americana de Doenças Infecciosas (IDSA) recomenda o tratamento da candidúria para: recém nascidos de muito baixo peso, pacientes neutropênicos, pacientes submetidos a transplante renal ou aqueles com indicação de manipulação invasiva ou cirúrgica de vias urinárias, mesmo na ausência de sintomas e obviamente, nos pacientes sintomáticos ${ }^{28}$; c) predisposto à candidúria com provável disseminação sistêmica: pacientes críticos com fatores de risco para infeção fúngica sistêmica e que evoluem com sinais de sepse e candidúria devem ser investigados para candidíase invasiva (hemoculturas) e iniciar o uso de antifúngico sistêmico ${ }^{28} 37$.

Qual esquema terapêutico a ser indicado? A terapêutica antifúngica para os casos de candidúria pode ser realizada com: anfotericina B sistêmica, anfotericina B tópica (irrigação vesical), fluconazol endovenoso ou fluconazol oral ${ }^{16} 2026$.

Apesar da boa atividade antifúngica da caspofungina e do voriconazol em espécies de Candida sp não há experiências clínicas relatadas na literatura. Dados pré-clínicos e de farmacocinética sugerem que as concentrações urinárias das equinocandinas sejam muito baixas ${ }^{1628}$.

0 uso isolado de 5-fluorocitosina é pouco recomendado pela possibilidade de surgimento de resistência durante a terapêutica ${ }^{28}$.

A experiência limitada do itraconazol na abordagem de pacientes com infecções invasivas por Candida, bem como os problemas de biodisponibilidade da sua formulação em cápsula, características farmacocinéticas de ligação protéica e sua reduzida excreção urinária não permitem a inclusão deste fármaco como opção terapêutica neste cenário ${ }^{16} 2026$.

Infecções urinárias causadas por leveduras sensíveis ao fluconazol devem ser tratadas com este triazólico. 0 fluconazol tem boa penetração no trato urinário, sendo que sua eficácia e tolerabilidade foram demonstradas em diferentes estudos. A dose recomendada varia de 100 a 400mg/dia (a maioria dos autores preconiza 100 a 200mg/dia), na dependência do agente envolvido, sua sensibilidade à droga e estado clínico do doente. Há esquemas diversos para utilização de fluconazol, sendo que o tempo de tratamento varia de 7 a 14 dias $^{19}$. Na suspeita de candidíase sistêmica, o paciente deverá ser tratado com doses de $400 \mathrm{mg} /$ dia e por período mínimo de 14 dias após melhora clínica e negativação das culturas ${ }^{7} 162028$.

Infecções urinárias por agentes resistentes ao fluconazol devem ser tratados com anfotericina B. 0 uso sistêmico deste poliênico é preconizado nos casos de suspeita de pielonefrite ou candidíase sistêmica, sendo recomendadas doses de $0,5 \mathrm{a} 1 \mathrm{mg} /$ kg dia, por ao menos 2 semanas, na dependência da doença de 
base do paciente, situação clínica e agente envolvido ${ }^{92}$. Casos de cistite fúngica por espécies ou isolados resistentes ao fluconazol podem ser tratados com irrigação vesical de anfotericina $B$, através da infusão diária de $50 \mathrm{mg}$ de anfotericina B diluída em água destilada, por período de 48 à $72 \mathrm{hs}^{5} 1628$.

Sobel e cols ${ }^{36}{ }^{37}$ realizaram estudo em 316 pacientes hospitalizados com candidúria (assintomáticos ou minimamente sintomáticos) para avaliar a eficácia e regime de fluconazol versus placebo por 14 dias. Observou-se neste estudo que a negativação da urina no $14^{\circ}$ dia ocorreu em $50 \%$ no grupo fluconazol e em $29 \%$ no grupo placebo. A depuração do fungo na urina também foi observada em $78 \%$ dos pacientes que receberam fluconazol por 14 dias e retiraram o cateter urinário contra $47 \%$ dos pacientes que receberam placebo por 14 dias e retiraram o cateter urinário. Vale mencionar que houve negativação da cultura em $35 \%$ dos pacientes que somente retiraram o cateter urinário. Durante o seguimento de 28 dias e duas semanas pós-tratamento, as taxas de erradicação da candidúria foram semelhantes nos dois grupos. Este estudo demonstra que o fluconazol é seguro, efetivo e produz altas taxas de erradicação microbiológica nas primeiras duas semanas e que a retirada do cateter é medida extremamente benéfica para que não haja recorrência da colonização e ou infecção ${ }^{36}$.

Outro estudo conduzido por Fan-Havard e cols ${ }^{5}$ comparando irrigação vesical com anfotericina $B$ versus fluconazol oral demonstrou negativação de culturas mais rápida com a irrigação vesical por anfotericina B. Entretanto, as taxas de erradicação com uma semana e com um mês após término da terapia não mostrou diferença entre os dois regimes terapêuticos 5 .

De forma geral, havendo indicação de terapêutica com antifúngico, a melhor opção é o uso de fluconazol na dose de 100-200mg/dia por 7 a 14 dias, tendo em vista sua eficácia, segurança e altos níveis de antifúngico encontrados na urina e no parênquima renal.

Quais os cuidados adicionais no manuseio clínico destes pacientes? Quando o médico decide que o paciente com candidúria deve ser submetido à terapêutica antifúngica há necessidade de se avaliar alguns aspectos adicionais: a) se houver risco clínico e epidemiológico da candidúria estar associada à candidíase hematogênica é fundamental a solicitação de hemoculturas para fungos, bem como a procura de complicações oftalmológicas de candidemia ${ }^{1728}$; b) pacientes em uso de sondagem vesical de demora devem ter o sistema retirado caso seja possível, logo que se inicie a terapêutica antifúngica. A persistência da sondagem vesical de demora em pacientes de risco ocasiona altas taxas de recidiva da candidúria (mais de 40\%) ${ }^{15}$; c) doenças obstrutivas do trato urinário devem ser investigadas em pacientes com candidúria persistente ou recorrente ${ }^{1728}$.

\section{CANDIDÚRIA EM PACIENTES SUBMETIDOS A TRANSPLANTE RENAL}

Candida é o gênero mais comum entre os agentes de infecção fúngica em pacientes transplantados renais. Embora tais infecções sejam, em sua maioria assintomática, elas podem levar ao desenvolvimento de pielonefrite e infecção disseminada. A epidemiologia da candidúria não é bem conhecida nesta população $0^{22} 34$.

Safdar e cols realizaram estudo caso-controle para determinar a evolução da candidúria em pacientes transplantados renais, durante o período de 1994 a 2001, identificando como casos pacientes com candidúria (uma amostra de urina com $>1.000$ UFC/mL de levedura) e os controles, pacientes sem candidúria. Foram analisados 1.738 pacientes submetidos a transplante renal, sendo que a candidúria ocorreu em 276 episódios encontrados em 192 (11\%) dos pacientes, sendo este grupo pareado com 192 controles sem esta complicação. A mediana de tempo da ocorrência da candidúria foi de 54 dias após o transplante. Candida glabrata correspondeu a 146 (53\%) dos fungos isolados, seguida da Candida albicans em 96 (35\%) dos casos. Com relação à sintomatologia, febre esteve presente em 25\% dos casos e sintomas genitourinários, em menos de $20 \%$ dos casos, sendo que $44 \%$ dos pacientes eram assintomáticos. Candidemia ocorreu em 10 (5\%) casos, com múltiplos fatores de risco para fungemia, incluindo a cateterização venosa central e a internação em unidade de terapia intensiva. A análise multivariada demonstrou os seguintes fatores de risco para aquisição de candidúria: sexo feminino, diabetes mellitus, bexiga neurogênica, desnutrição, uso prévio de antimicrobianos, presença de cateter vesical, presença de stents e admissão na UTI. 0 uso prévio de fluconazol não foi fator protetor para a ocorrência de candidúria ${ }^{33}$.

A análise de mortalidade em 90 dias destes pacientes revelou um total de 64 óbitos, sendo 16 (8\%) no grupo controle e 48 (25\%) no grupo com candidúria. A única causa de óbito relacionada à infecção fúngica foi aspergilose invasiva ${ }^{33}$.

Neste universo, a administração de antifúngicos foi realizada em 97 pacientes, sendo 59 (61\%) com fluconazol e 58 (60\%) com irrigação vesical com anfotericina B. Um total de 95 pacientes não foi tratado com antifúngicos e 119 (62\%) retiraram o cateter vesical. 0 grupo tratado teve 32 mortes e o grupo não tratado, 16 mortes $^{33}$.

Apesar de algumas limitações do estudo, o tratamento da candidúria não foi preditor independente na taxa de sobrevida nem resultou em maior probabilidade de esterilização da urina em pacientes submetidos a transplante renal ${ }^{33}$. Apesar da recomendação do guia da IDSA que preconiza o tratamento de candidúria em transplantados renais mesmo que assintomáticos, pelo risco da perda do enxerto, observa-se que esta recomendação tem níveis de evidência científica muito limitados. 0 estudo de Safdar mostrou claramente que a terapêutica com fluconazol em pacientes com candidúria e transplante renal teve benefícios marginais frente ao grupo não tratado ${ }^{28} 33$. Neste contexto, talvez seja mais adequado restringir a utilização de antifúngicos em pacientes com candidúria sob maior risco de perda do enxerto e/ou evolução para doença invasiva disseminada, a exemplo de pacientes nos primeiros três meses após o transplante renal, em casos de suspeita de doença invasiva, usuários de altas doses de imunossupressores e submissão a procedimentos cirúrgicos ou manipulação do trato urinário 2233 . 


\section{REFERÊNCIAS}

1. Alvarez-Lerma F, Nolla-Salas J, Leon C, Palomar M, Jorda R, Carrasco N, Bobillo F. Candiduria in critically ill patients admitted to intensive care medical units. Intensive Care Medicine 29: 1069-1076, 2003.

2. Ang BSP, Telenti A, King B, Steckalberg JM, Wilson WD. Candidemia from urinary tract source: microbiological aspects and clinical significance. Clinical Infectious Diseases 17: 622-627, 1993.

3. Binelli CA, Moretti ML, Assis RS, Sauaia N, Menezes PR, Ribeiro E, Geiger DCP, Mikami Y, Miyaji M, Oliveira MS, Barone AA, Levin AS. Investigation of the possible association between nosocomial candiduria and candidaemia. Clinical Microbiology and Infection 12: 538-543, 2006.

4. Colombo AL, Barchiesi F, McGough DA, Rinaldi MG. Comparison of E-test and NCCLS macrodilution method for azole antifungal susceptibility testing. Journal of Clinical Microbiology 33: 535-540, 1995.

5. Fan-Havard P, O'Donovan C, Smith SM, Oh J, Bamberger M, Eng RHK. Oral fluconazole versus amphotericin B bladder irrigation for treatment of candidal funguria. Clinical Infectious Diseases 21: 960-965, 1995.

6. Febré N, Silva N, Medeiros EAS, Wey SB, Colombo AL, Fischmann O. Microbiological characteristics of yeasts isolated from urinary tracts of intensive care unit patients undergoing urinary catheterization. Journal of Clinical Microbiology 37: 15841586,1999

7. Fisher JF. Candiduria: when and how to treat it. Current Infectious Diseases Report 2: $523-530,2000$

8. Fisher JF, Newmam CL, Sobel JD. Yeasts in the urine: solutions for a budding problem. Clinical Infectious Diseases 20: 183-189, 1995.

9. Fisher JF, Woeltje K, Espinel-Ingroff A, Stanfield J, DiPiro JT. Efficacy of a single intravenous dose of amphotericin B for Candida urinary tract infections: further favorable experience. Clinical Microbiology and Infection 9: 1024-1027, 2003.

10. Garner JS, Jarvis WR, Emori TG, Horan TC, Hughes JM. Centers for Diseases Control and Prevention: definitions for nosocomial infections. American Journal of Infection Control 16: 128-140, 1988

11. Gubbins PO, Piscitelli SC, Danziger LH. Candidal urinary tract infections: a comprehensive review of their diagnosis and management. Pharmacotherapy 13: 110-127, 1993

12. Harmory BH, Wenzel RP. Hospital-associated candiduria predisposing factors and review of the literature. Journal of Urology 120: 444-448, 1978.

13. Hosphental DR, Murray CK, Rinaldi MG. The role of antifungal susceptibility testing in the therapy of candidiasis. Diagnostic Microbiology and Infectious Diseases 48: 153-160, 2004

14. Jarvis WR. Epidemiology of nosocomial fungal infections with emphasis on Candida species. Clinical Infectious Diseases 20: 156-160, 1995.

15. Johnson JR. Should all catheterized patients with candiduria be treated? Clinical Infectious Diseases 17: 814, 1993

16. Kauffman CA. Candiduria. Clinical Infectious Diseases 41: S371-376, 2005.

17. Kauffman CA, Vazquez JA, Sobel JD. Prospective multicenter surveillance study of funguria in hospitalized patients. Clinical Infectious Diseases 30: 14-18, 2000.

18. Kobayashi CC, Fernandes OF, Miranda KC, Sousa ED, Silva MR. Candiduria in hospital patients: a study prospective. Mycopathologia 158: 49-52, 2004.

19. Leu H-S, Huang C-T. Clearance of funguria with short-course antifungal regimens: a prospective, randomized controlled study. Clinical Infectious Diseases 20: $1152-1157,1995$.

20. Lundstrom T, Sobel J. Nosocomial candiduria: a review. Clinical Infectious Diseases 32: 1602-1607, 2001.
21. Magill SS, Swoboda SM, Johnson EA, Merz WG, Pelz RK, Lipsett PA, Hendrix $\mathrm{CW}$. The association between anatomic site of Candida colonization, invasive candidiasis and mortality in critically ill surgical patients. Diagnostic Microbiology Infectious Diseases 55: 293- 301, 2006.

22. Moysés Neto M, Costa RS, Reis MA, Garcia TMP, Ferraz AS, Saber LTS, Batista MEPN, Muglia V, Figueiredo JFC. Candidíase em pacientes transplantados renais. Revista da Sociedade Brasileira de Medicina Tropical 30: 485-491, 1997.

23. Nassoura Z, Ivatury RR, Simon RJ, Jabbour N, Stahl WMI. Candiduria as an early marker of disseminated infection in critically ill surgical patients: role of fluconazole therapy. Journal of Trauma 35: 290-295, 1993.

24. National Committee for Clinical Laboratory Standards. Reference method for broth dilution antifungal susceptibility testing of yeasts. NCCLS document M-27 A2. Wayne PA, 2002.

25. Navarro EE, Almario JS, Schaufele RR, Bacher J, Walsh TJ. Quantitative urine cultures do not reliably detect renal candidiasis in rabbits. Journal of Clinical Microbiology 35: 3292-3297, 1997.

26. Nucci M. Candiduria in hospitalized patients: a review. Brazilian Journal of Infectious Diseases 4: 168-172, 2000.

27. Oliveira RD, Maffei CM, Martinez R. Nosocomial urinary tract infections by Candida species. Revista da Associação Médica Brasileira 47: 231-235, 2001.

28. Pappas PG, Rex JH, Sobel JD, Filler SG, Dismukes WE, Walsh TJ, Edwards JE. Guidelines for treatment of candidiasis. Clinical Infectious Diseases 38: 161-189, 2004 .

29. Passos XS, Sales WS, Maciel PJ, Costa CR, Miranda KC, Lemos JA, Batista MA, Silva MRR. Candida colonization in intensive care unit patients' urine. Memórias do Instituto Oswaldo Cruz 100: 925-928, 2005.

30. Pfaller MA. Nosocomial candidiasis: emerging species, reservoirs and modes of transmission. Clinical Infectious Diseases 22: 89-94, 1996.

31. Rex JH, Pfaller MA, Galgiani JN. Development of interpretative breakpoints for antifungal susceptibility testing: concept framework and analysis of in vivo - in vitro correlation data for fluconazole, itraconazole and Candida infections. Clinical Infectious Diseases 24: 235-247, 1997.

32. Rex JH, Rinaldi MG, Pfaller MA. Resistance of Candida species to fluconazole. Antimicrobial Agents and Chemotherapy 39: 1-8, 1995.

33. Safdar JD, Slattery WR, Hnasinski V, Gangnon E, Li Z, Pirsch JD, Andes D. Predictors and outcomes of candiduria in renal transplant recipients. Clinical Infectious Diseases 40: 1413-1421, 2005.

34. Singh N. Fungal infections in the recipients of solid organ transplantation. Infectious Diseases of Clinical North America 17:113-134, 2003.

35. Sobel JD. Controversies in the diagnosis of candiduria: what is the critical colony count. Current Treatment Options Infectious Diseases 4: 81-83, 2002.

36. Sobel JD, Kauffman CA, McKinsey D, Zervos M, Vazquez JA, Karchmer AW, Lee J, Thomas C, Panzer H, Dismukes WE. Candiduria: a randomized, double-blind study of treatment with fluconazole and placebo. Clinical Infectious Diseases 30: $19-24,2000$.

37. Sobel JD, Vazquez JA. Fungal infections of the urinary tract. World Journal of Urology 17: 410-414, 1999.

38. Vandenbossche I, Vaneechoutte M, Vandevenne M, Baere T, Verschraegen G Susceptibility testing of fluconazole by NCCLS broth macrodilution method, E-test and disk diffusion for application in the routine laboratory. Journal of Clinical Microbiology 40: 918-921, 2002.

39. Weinbergger M, Sweet S, Leibivici L, Pitlik SD, Samra Z. Correlation between candiduria and departmental antibiotic use. Journal of Hospital Infection 53 : 183-186, 2003. 